\title{
Granular processes on Itokawa, a small near-Earth asteroid: implications for resource utilization
}

Hideaki Miyamoto, Jeffrey S. Kargel, Wolfgang Fink, Roberto Furfaro

Hideaki Miyamoto, Jeffrey S. Kargel, Wolfgang Fink, Roberto Furfaro, "Granular processes on Itokawa, a small near-Earth asteroid: implications for resource utilization," Proc. SPIE 6960, Space Exploration Technologies, 69600I (15 April 2008); doi: 10.1117/12.784634

Event: SPIE Defense and Security Symposium, 2008, Orlando, Florida, United States 
Invited Paper

\title{
Granular processes on Itokawa, a small near-Earth asteroid: Implications for resource utilization
}

\author{
Hideaki Miyamoto*a, Jeffrey S. Kargel ${ }^{b}$, Wolfgang Fink ${ }^{\mathrm{c}}$, Roberto Furfaro ${ }^{\mathrm{d}}$ \\ ${ }^{a}$ University Museum, University of Tokyo, Tokyo, Japan \\ ${ }^{\mathrm{b}}$ Lunar and Planetary Laboratory, University of Arizona, Tucson, AZ, USA \\ ${ }^{\circ}$ California Institute of Technology, Visual and Autonomous Exploration Systems Research \\ Laboratory, Division of Physics, Mathematics \& Astronomy, Pasadena, CA, USA \\ ${ }^{\mathrm{d}}$ Aerospace and Mechanical Engineering Department, University of Arizona, Tucson, AZ
}

\begin{abstract}
Crucial questions for possible utilization of Near Earth Asteroids include how to break asteroid materials down to particle sizes that can be processed. This remained difficult to answer because of the limited number and resolutions of images previous obtained through asteroid missions. Recently, the Hayabusa spacecraft obtained unprecedentedly highresolution images of a $\sim 300 \mathrm{~m}$-sized asteroid, Itokawa, which gives unique opportunity to discuss the nature of surface materials on a small asteroid. Hayabusa reveals that the asteroid is covered by fine- and coarse-grained materials, including granules, pebbles, cobbles, and boulders up to tens of meters. Gravels on this small asteroid appear to be loosely deposited along the gravitational equipotential surfaces. The existence of smooth areas as well as boulder-rich rough areas indicate that gravels should have experienced migrations and segregations. Thus, the issue regarding the breaking of asteroid materials appears to have been resolved naturally, at least for this asteroid, which has important implications for future robotic missions dedicated to resource exploration and utilization.
\end{abstract}

Keywords: regolith, asteroid, space resources, space exploration, robotic mining operation

\section{INTRODUCTION}

Near Earth Asteroids are scientifically fascinating targets because the majority of NEAs are believed to be derived from the main asteroid region as collisional fragments of rock and/or metal from parent asteroids, indicating that NEAs hold keys to understanding the formation and evolution of the Solar System. Recently growing another incentive to study the NEAs includes potential utilizations of the resources, because the NEAs are the nearest bodies to Earth. Based on studies of meteorites, most of which are derived from NEAs, we have fairly solid evidence that the potential resources of these objects are diverse and may hold the keys to the industrialization and settlement of the inner Solar System (Lewis 1993). However, evaluating the economic usefulness of asteroids is difficult because many crucial questions are unanswered, including the accessibility to resources of asteroids. Other than technical difficulties of getting to and returning from asteroids, there is the issue of breaking asteroid materials down to particle sizes that can be processed. This latter issue appears to have been resolved naturally, at least for one asteroid, by virtue of the unexpected discovery that Itokawa possesses a regolith.

Breaking asteroid materials is a part of natural processes of the formation of regolith, which is generally defined as any kind of superficial layer/blanket of loose particulate rock materials. The existence of regolith would significantly reduce the energy for utilizing materials on asteroids, and thus is an important question in terms of future resource utilization. Regolith materials are expected to form when meteoroids impact onto the asteroid surface. Although this happens regardless the size of asteroids, a small body may lack in regolith materials due to its lower escape velocity. Also, the existence of regolith may change the characteristics of reflectance, which becomes important issue to evaluate chemical properties of asteroids from terrestrial observations. Therefore, whether smaller asteroids are covered by regolith or not

Space Exploration Technologies, edited by Wolfgang Fink

Proc. of SPIE Vol. 6960, 69600I, (2008) · 0277-786X/08/\$18 - doi: 10.1117/12.784634 

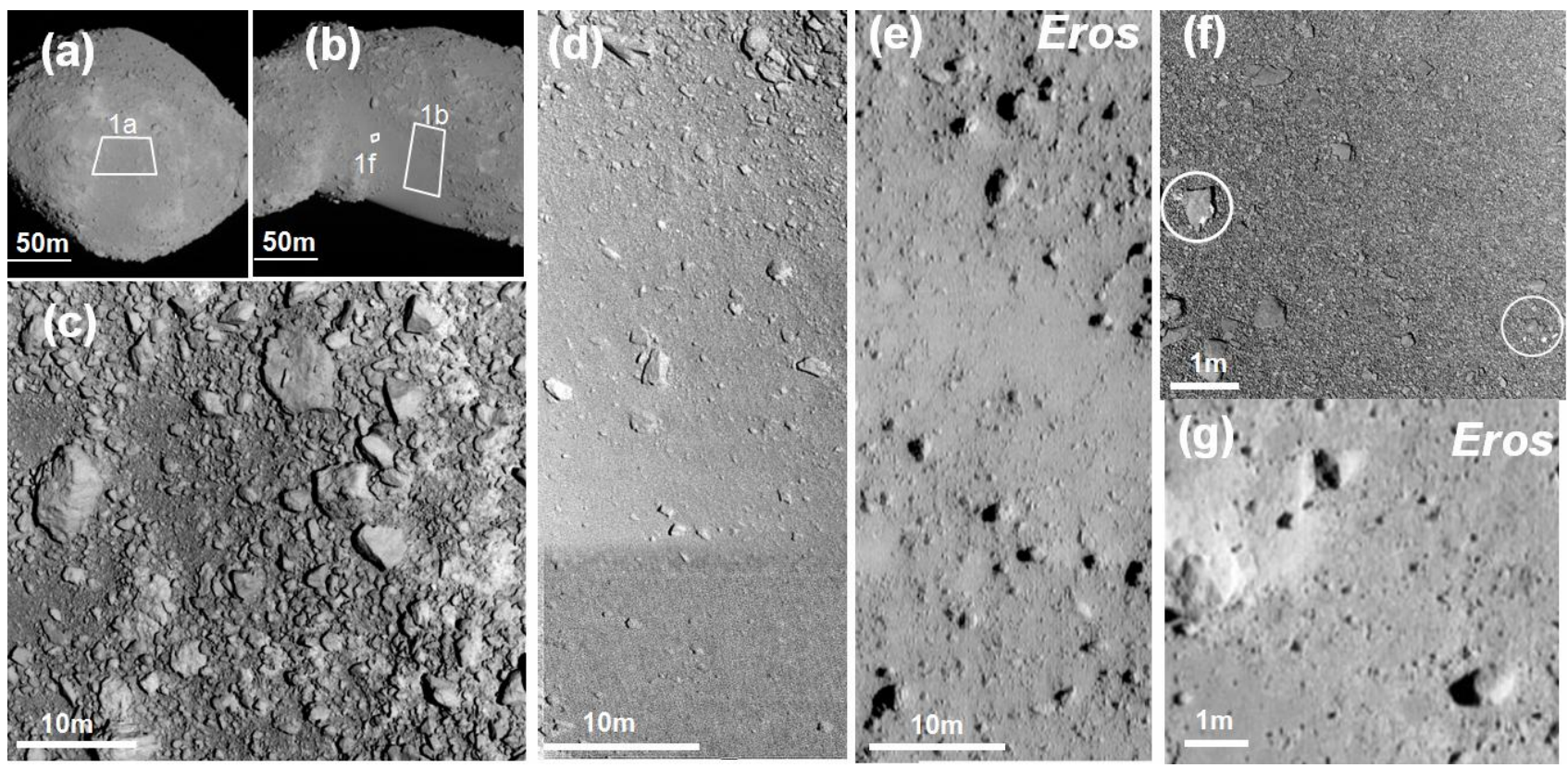

Fig. 1. Images show the different appearances with different resolutions. In the images at the Home Position $(\sim 7 \mathrm{~km})$, we identified several smooth areas including Little Woomera (a; ST_2424189738) and Muses Sea (b; ST_2451105131), whose appearances are similar in terms of surface smoothness, brightness, and even color. However, the higher resolution images obtained at an altitude of $\sim 600 \mathrm{~m}$ reveal that the sizes of particles forming the smooth appearance are different; while Muses Sea (d; ST_2532629277) appears to be covered by fines, whose grain size is not resolved in this resolution, Little Woomera (c; ST 2530297837 ) is covered by numerous gravels, whose sizes are mostly resolved (ranges from less than a meter to more than 5 meters). The appearance of Muses Sea at this distance is quite similar to the pond deposits of Eros (e), but not in the high resolution images (f; ST_2563511720) compared with similar resolution image of pond deposits of Eros (g). Circles in (f) indicate bright pebbles.

was a significant unanswered question both for understanding evolutions of asteroids and for evaluating possibilities of utilizing surface resources on asteroids.

In terms of space resource, the existence of regolith on a small body might have higher importance than those on a larger body, because a small body has a small surface gravity and, thus, smaller delta-v, which is economically beneficial especially when returning objects from the surface. However, the presence of regolith, especially on a small body, is technically difficult to directly confirm with optical or thermal observations from the ground due to poor spatial resolutions for small objects. On this ground, images of Itokawa taken by the Hayabusa spacecraft give the unique opportunity to study the nature of regolith materials on a sub-km sized asteroid. Itokawa is an S-type and the smallest asteroid (550m x 300m x 240m) yet observed by spacecraft (Fujiwara et al, 2005).

\section{HAYABUSA'S OBSERVATIONS}

The shape of Itokawa is not simple; it appears to be composed of a smaller angular part (the "Head") and a larger, rounded part (the "Body") (Fujiwara et al., 2006). The surface of this asteroid is covered by unexpectedly large number of boulders. Their distribution is not uniform, which allows classifying two terrain types, such as rough and smooth terrains (Saito et al, 2006).

In low resolution images before Hayabusa closely approach to Itokawa, Itokawa appears to be somehow similar to previously-studied S-type asteroids. In images taken at distance (Figure 1a and 1b), rough terrains are observed to dominate most of the area of Itokawa, and smooth terrains fill in certain areas. The rough terrains generally show a similar appearance with numerous boulders that mostly appear to be buried in smooth, homogeneous materials as often observed for other S-type asteroids previously studied by space missions. This gives an impression that boulders may have been completely buried by fine materials. Contrary to this, the first detailed observations of Itokawa by the 
Hayabusa spacecraft reveal that the appearance of the regolith on this tiny asteroid is surprisingly different from any other previously-observed asteroids (Figure 1).

The nature of these terrains on Itokawa are best illuminated by images at the ranges lower than $2 \mathrm{~km}$ obtained during the descent phase of the mission for specific purposes, including the sampling (Yano et al., 2006). Muses-Sea smooth terrain was most frequently imaged, especially because it was selected as the touch-down site for the Hayabusa spacecraft due mostly to technical reasons, which favour the its most distinctive smoothness. The Muses Sea is located between these two parts as if the terrain fills the gap of the contact area (Figure 2). Therefore in this sense, there was originally a larger depression much deeper than it appears now. However, we observe the significantly evolved, non-random distribution of granular materials in the highest resolution images, which would require additional explanations.

We found that this area was covered by well-sorted particles (Figure 1f). Although the images cannot completely resolve the particles' shapes, the textures of the images are typically composed of a combination of both a few to several brighter pixels and one to a few dark pixels. Considering the highest resolution (about $6 \mathrm{~mm}$ per pixel) and the sun-angle (about 23 degree, calculated by a numerical shape model), the grain sizes of fine particles likely range from sub-centimetre to centimetre-scale, which is much coarser grains than those of the "ponds" on Eros. If much finer materials were accumulated, more coverage by much smoother deposits would have been observed in these high-resolution images.

All of close-up images of Itokawa show that gravels are typically piled on each other without being buried by fines. The nature of gravel piles is most vividly suggested by the collapse of a rim-like feature of a possible crater (Figure 2). From these close-up images (Figures 3 and 4), we can infer that the surface of Itokawa is likely covered by unconsolidated gravel.

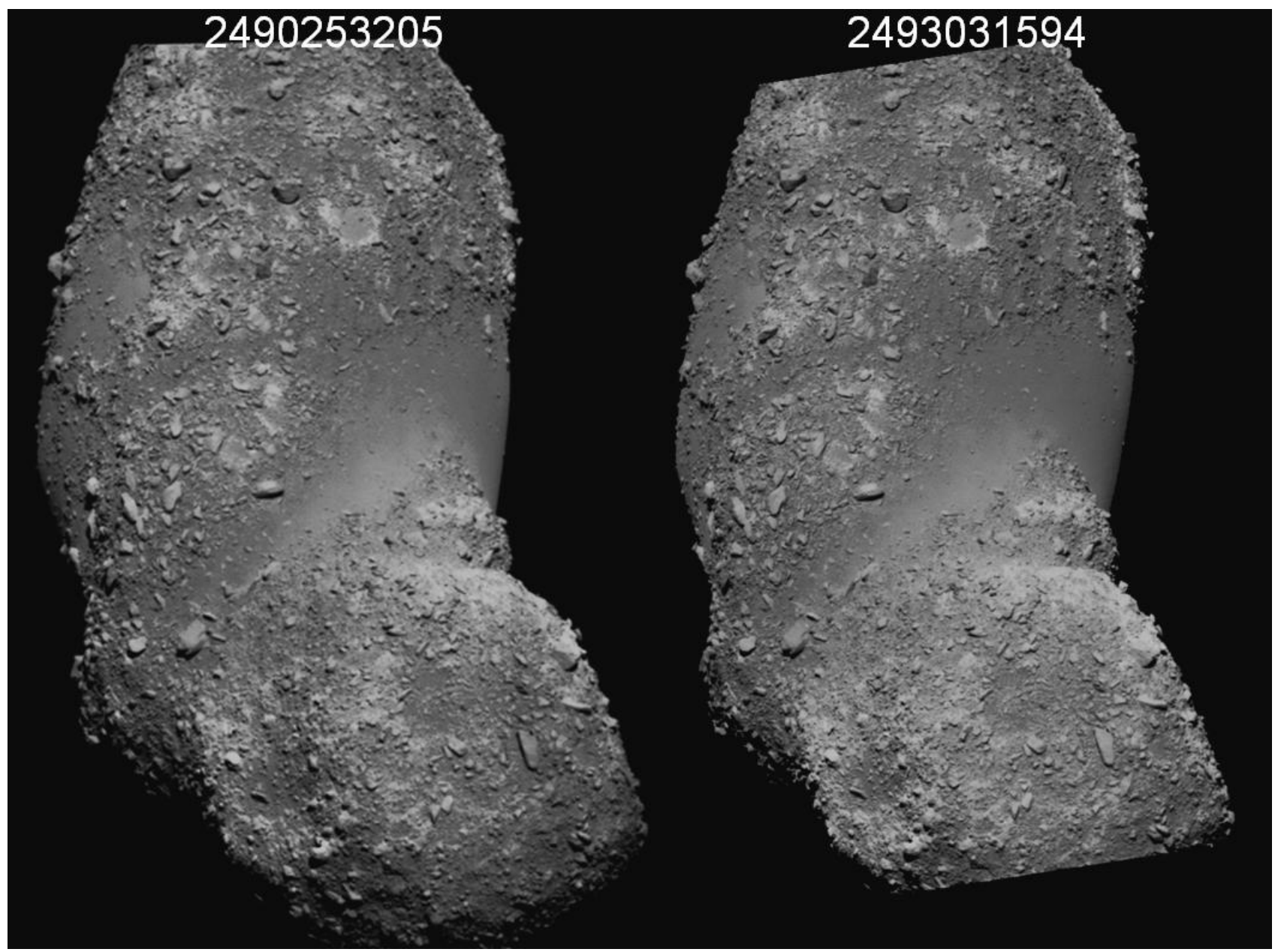

Fig. 2. Stereo pair of Itokawa showing the Muses-C region, which appears to be located at the concave region between the Head (lower part) and the Body (upper part) of this asteroid. 


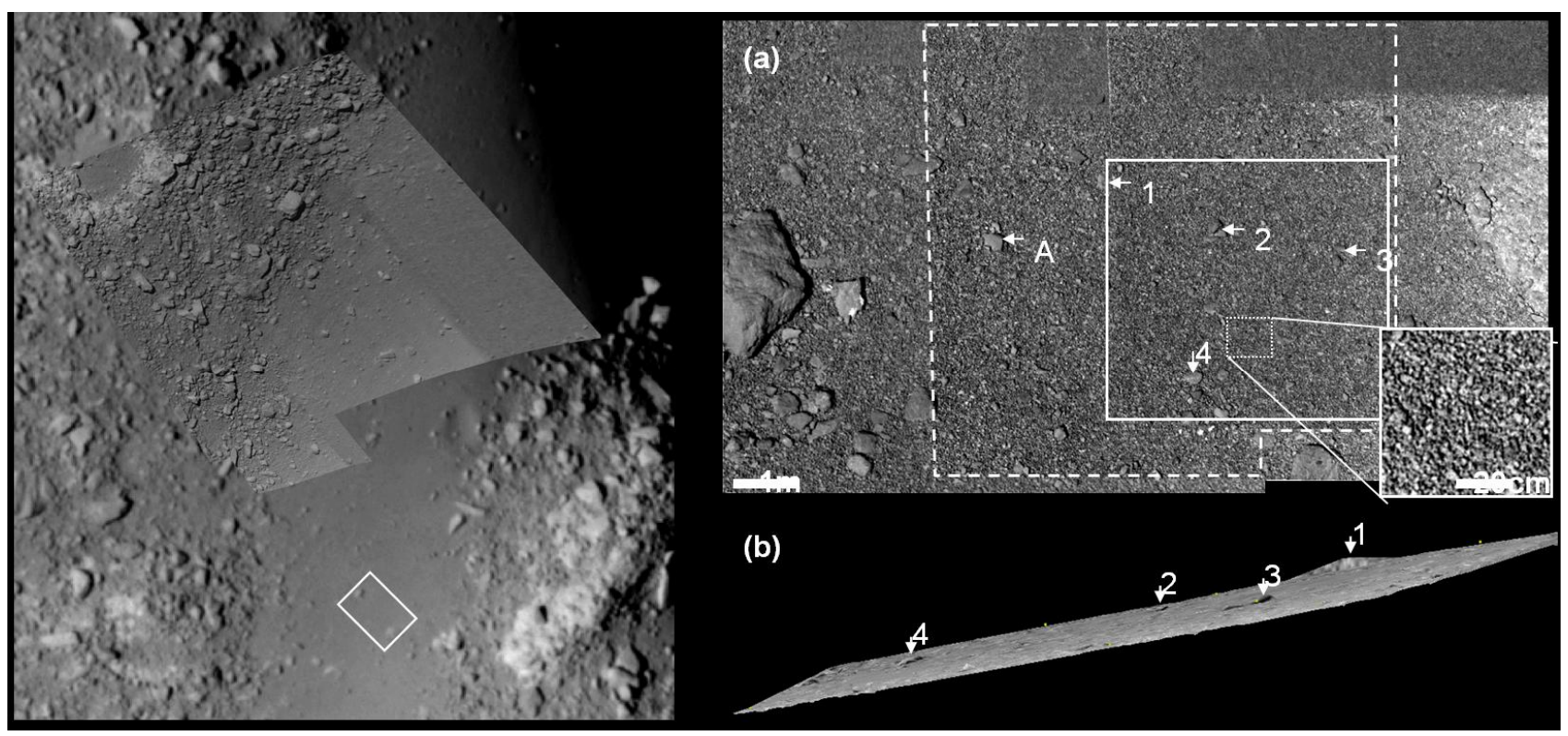

Fig. 3. (Left) Muses-Sea region. White box indicates the location of the close-up images shown in the figure on the right. (right) (a) Mosaic of the highest resolution images at the Muses Sea (ST2559934516, ST2559938356, and ST2559940276). Dotted area is overlapped by at least two images, which allows detailed stereo analyses as presented in Miyamoto et al (2007). Inset is an enlarged view of the area shown in the broken line, showing that this area is covered by millimeter-centimeter sized pebbles. White box shows the area graphically plotted in (b). Note that this area is topographically flat almost completely and is covered by fine particles, whose sizes are likely a few centimeters. Most boulders appear to be placed on the surface of fine particles, especially because they are not buried even partially on their sides. (b) Oblique view of the digital elevation model derived from the numerical stereo analyses with more than 11,000 control points using the StereoEyeV3 software.

\section{REGOLITH ON SMALL ASTEROIDS}

\subsection{Previous views}

The formation of regolith on the Moon or other larger bodies is likely different from that on the asteroids, due to the significant difference in surface accelerations; While impact ejecta are locally deposited around craters on the Moon, on an asteroid they are easy to spread balistically over the entire surface and often escape. An old view, now invalidated by the observations made by Hayabusa, is that asteroids below some small size, such as Itokawa, would be completely devoid of regolith due to low gravitational energy. Also, the regolith on an asteroid is believed to be globally continuous, poorly-sorted, because repetitious impacts on asteroids easily form ballistically distributed ejecta deposits due to low gravity. This view, in fact, plausibly explains the generally uniform appearances of S-type asteroids previously explored by spacecraft.

The existence of globally-segregated smooth terrains on Itokawa requires additional explanation than this previous view. Although repetitious impacts on the Moon form locally concentrated, size-sorted regolith, this process does not work to form locally-distinctive deposits on a small asteroid. One plausible explanation for this is the migration and segregation of surface materials by vibrations due likely to impacts (Miyamoto et al., 2007). 


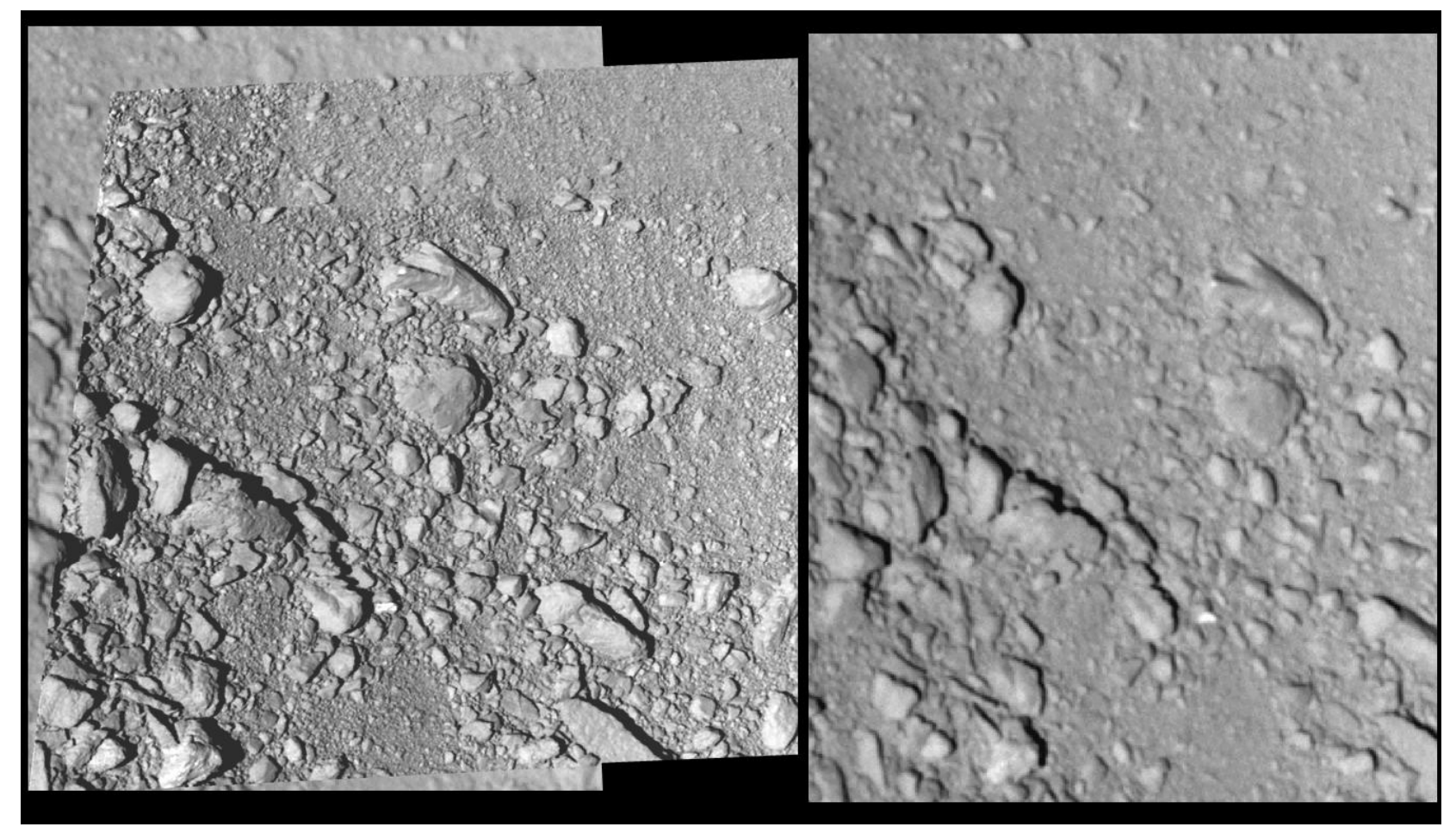

Fig. 4. Two different-resolution images at the boundar area between Muses sea and rough terrain. Using as stereo images (high-resolution, ST2539423137, as left and medium resolution, ST2532629277, as right), geomorphological evidence for gravel migrations can be clearly seen, including boulder alighments and partial ponding of smaller gravels on the upstream-sdelarger boulders.

\subsection{Regolith migrations}

Regolith migrations and segregations are proposed by Miyamoto et al. (2007) to explain the characteristics of gravels on Itokawa observed by the Hayabusa spacecraft. A short review of the idea is the following: On the smooth terrains on Itokawa, the slope angles against the local gravity are generally lower than 8 degrees. This might be indicative that the smooth terrains are filled by materials that are fluidized and migrated along the local gravity. Also, Muses Sea is estimated to be the minimum over the entire surface of Itokawa. These may imply the gravitationally-induced movements of the finer materials after the formation of the asteroid. Smooth terrains, including the largest smooth terrain (Sagamihara sea), always locate at the local lows of gravity, however, the local lows of gravity do not always have smooth deposits. These strongly indicate that there should have gravity-driven migrations of regolith on the surface of Itokawa.

In fact, the regolith migration on asteroid surfaces has been suggested previously, from slope processes to a looselydefined "reshuffling" of the surface. However, because the motion indicators that were considered are mostly indirect (such as the scars of slope-processes), the nature and significance of regolith migration remained unclear. The pond deposits on Eros clearly indicate the secondary migrations of fines, which may be explained by either electrostatic levitation or the redistribution of fines during impact-induced shaking. However, due to a lack of data at sufficient resolution both explanations still remain viable.

The pond deposits on Eros appear similar to the smooth terrains on Itokawa in the images taken at distance (Figure 1a). However, close-up images (Figure 1f) reveal that the smooth terrain is covered by well-sorted pebbles, that are too coarse to be transported by electrostatic levitation, implying its minor role (except perhaps for transporting or loosing much finer particles). The high mobility of the regolith materials is also supported by the fact that both poles are covered by smooth terrains. Especially, the location of the north-pole exactly matches the apex of the central summit of the dome-like shape in Sagamihara. This is plausible because the surface on the rotational axis would be gravitationally low, particles with higher mobility would have been deposited. 


\section{DISCUSSIONS}

The Hayabusa spacecraft is the first mission that observed the surface conditions of a small, $\sim 300 \mathrm{~m}$-sized asteroid in high resolutions. The landing operations were the optimal chance to obtain high-resolution images of the surface, which revealed that Itokawa is covered by fine- and coarse-grained materials from granules to boulders up to tens of meters. This is completely different from the previous views of small asteroids, which were believed to have no regolith. The appearance of Itokawa is of a so-called "rubble pile," but poorly understood sorting processes have acted to redistribute materials according to grain size.

We consider that, because the size of Itokawa is by far the smallest asteroid ever studied at high resolution (e.g., the mass of Itokawa is six orders of magnitudes smaller than Ida, which is six orders of magnitudes smaller than the Moon), the sorting processes of regolith active on Itokawa has not seen before. We propose that the regolith, accumulated as a consequence of a rubble pile asteroid, segregated as a result of their global migrations (Miyamoto et al., 2007). The fluidity is believed to be triggered by impact-induced vibrations, though the vibrations could be caused by several other factors, such as tidal effects, thermal fluctuations, or low-speed collisions between the Head and Body. Nevertheless, it might be helpful to consider the seismic effect of impacts to discuss that it is easier for a smaller asteroid to be shaken at sufficiently large accelerations that can destabilize materials.

If we follow the theory presented by Richardson et al., the average seismic strain energy per unit volume, $e$, of rock that is vibrating at the maximum half-cycle amplitude, $A$, and the seismic frequency, $f$, may be written as:

$e=\pi^{2} \rho_{a} f^{2} A^{2}$

where $\rho_{a}$ is the bulk density of the asteroid. The total seismic energy density in the system e may be given as $e=2 e_{d}$. In this case, the total amount of seismic energy, $E_{s}$, of the asteroid whose radius is $D_{a}$ is thus given as:

$E_{s}=\frac{\rho_{a} D_{a}^{3} a^{2}}{48 \pi f^{2}}$

Assuming that the seismic energy is completely supplied by the kinetic energy of an impactor $E_{i}$, we obtain the maximum acceleration caused by an impact as

$a=2 \pi v_{i} f \sqrt{\eta\left(\frac{\rho_{i}}{\rho_{a}}\right)\left(\frac{D_{i}}{D_{a}}\right)^{3}}$.

, where $\eta$ is the seismic efficiency factor, $\rho_{i}$ is the bulk density of the impactor, $D_{i}$ is the radius of the impactor, and $v_{i}$ is the velocity of the impactor. Because the gravitational acceleration at the surface of the asteroid, $g$, is given as

$g=\frac{2}{3} \pi G D_{a} \rho_{a}$

we obtain the ratio of the maximum acceleration and the surface gravity, $a / g$, as

$$
\frac{a}{g}=\frac{3 f v_{i}}{G} \sqrt{\eta \frac{\rho_{i}}{\rho_{a}^{3}} \frac{D_{i}^{3}}{D_{a}^{5}}},
$$

which is used to plot the dotted lines in Figure 5. Figure 5 shows that a/g becomes larger when the mean diameter of asteroid is smaller. In other words, a smaller asteroid is easier to have a higher a/g to destabilize the gravels on the surface. Laboratory experiments indicate that the migrations of particles can occur at a/g $\sim 0.2$, which is plotted as shadow in Figure 5. Thus, gravels on a smaller asteroid might be easy to migrate if certain amount of energy is given to the asteroid by impact. 


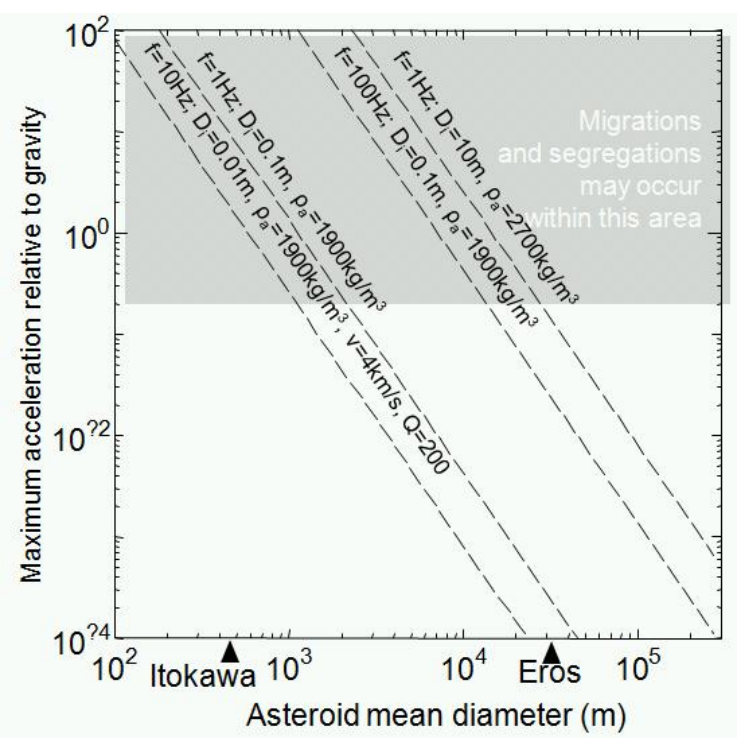

Fig. 5. The ratio of the maximum acceleration due to impact and the surface gravity against the asteroid mean diameter. We adopt the parameter values mostly the same as those by Richardson et al. (2005): $\mathrm{G}=6.67259 \times 10^{-11} \mathrm{~m}^{3} \mathrm{~s}^{-2} \mathrm{~kg}^{-1}$, $\mathrm{v}_{\mathrm{i}}=4 \mathrm{kms}^{-1}$ or $5 \mathrm{kms}^{-1}, \rho_{\mathrm{a}}=1900 \mathrm{kgm}^{-3}$ (similar to Itokawa) or $2700 \mathrm{kgm}^{-3}$ (similar to Eros), $\rho_{\mathrm{i}}=2500 \mathrm{kgm}^{-3}, \eta=10^{-4}, \mathrm{f}=1,10$, or $100 \mathrm{~Hz}, \mathrm{~K}=0.250 \mathrm{~km}^{2} \mathrm{~s}^{-1}$, and $\mathrm{Q}=200$ or 2000 . See Miyamoto et al. (2007) for more detail.

On Itokawa, we know that segregations can occur, which may be plausibly explained by the above discussions. If this is the case, it is a significant finding that the economic usefulness of asteroids is a function of the cost of getting to and returning from Earth, which is expected to be minimal for small asteroids. We note that many valuable substances, such as native metals, are abundant in meteoritic and asteroidal materials. Thus, if the migrations (and ultimately segregations) of gravels occur, these might enhance the enrichments of useful resources on the surface of small asteroids. Another important issue regarding utilization of regolith materials on asteroids is whether the regolith is loose or compacted/cemented. We found that, at least on Itokawa, unconsolidated gravels exist, which indicates that smaller asteroids might be enriched in accessible resources, that are naturally crashed.

Despite surface gravity verging near 0.01 milli-gee, Itokawa not only retains particulate material, but there is evidence of gravitational sorting based on grain size and possible downslope regolith migration. This fact has significant implications for future mining of small asteroids, for example: (1) A rubble-pile asteroid's low strength would suggest that it may require a very careful approach to move such asteroids in their entirety to other orbits. (2) If Itokawa is similar to other small asteroids, it should be possible to find large boulders and transport them in their entirety into low Earth orbit for use in space manufacturing. For example, a 25-m-wide block, such as one of the larger boulders identified on Itokawa, may have a mass of order 50,000 metric tons. This is 200 times the mass of the International Space Station, thus suggesting the possibility of some major space construction projects utilizing such a boulder's mass. If it were a carbonaceous chondrite type of asteroid instead of an S-type, this mass could contain anywhere from 1500 to 7000 tons of water and 500 to 1500 tons of carbon, and thousands of tons of many potentially useful metals and oxides. (3) Some asteroids may be highly heterogeneous chemically and mineralogically; this heterogeneity provides both a possible resource bonanza of varied materials as well as an exploration challenge. To meet this challenge, a robotic exploration campaign would be required (see other papers this volume by Fink et al., Furfaro et al., and Kargel et al.). To meet this challenge, a robotic exploration campaign would be required (see Fink et al. 2005, 2006a, 2007, 2008, 2006b, as well as other papers in this volume by Fink et al., Furfaro et al., and Kargel et al).

\section{References}

1. Lewis, J.S. (Ed.), 1993, Resources of Near Earth space, university of Arizona Press, Tucson.

2. Fink, W., et al., 2005, Next-Generation Robotic Planetary Reconnaissance Missions: A Paradigm Shift; Planetary and Space Science, 53, 1419-1426. 
3. Fink, W., et al., 2006a, Autonomous Tier-Scalable Reconnaissance Missions For Remote Planetary Exploration; Proceedings of the 4th International Planetary Probe Workshop 2006, Pasadena.

4. Fink, W., et al., 2007, Tier-Scalable Reconnaissance Missions For The Autonomous Exploration Of Planetary Bodies; IEEE Aerospace Conference Proceedings, Big Sky, Montana.

5. Fink, W., et al., 2008, Automated Global Feature Analyzer (AGFA) - A Driver for Tier-Scalable Reconnaissance, IEEE Aerospace Conference Proceedings, Big Sky, Montana.

6. Fink, W., 2006b, Generic Prioritization Framework for Target Selection and Instrument Usage for Reconnaissance Mission Autonomy, Proceedings of IEEE World Congress on Computational Intelligence (WCCI) 2006, Vancouver, Canada, 11116-11119.

7. A. Fujiwara et al., 2006, The Near-Earth Asteroid Itokawa observed by Hayabusa: Its Possible Structure and History,

Science, 312, 1330-1334.

8. Kargel, J.S., et al., this issue, Robotic Resource Exploration is a Key to Human Expansion Through the Cosmos, Paper accepted at the SPIE Space Explor. Technol. Conf. March 2008 in Orlando, FL.

9. R. Furfaro, J. Lunine, J. S. Kargel, W. Fink, Intelligent systems for the autonomous exploration of Titan, Enceladus., 2008. Paper accepted at the SPIE Space Explor. Technol. Conf. March 2008 in Orlando, FL.

10. R. Furfaro, J. Lunine, A. Elfes, K. Reh, Wind-based navigation of a hot-air balloon on Titan: a feasibility study, 2008. Paper accepted at the SPIE Space Explor. Technol. Conf. March 2008 in Orlando, FL.

11. Miyamoto, H., et al., 2007, Regolith Migration and Sorting on Asteroid Itokawa, Science, 316, 1011-1014, 2007.

12. Saito, H., et al., 2006, A first look at asteroid 25143 Itokawa: Observations by the multiband imager of HAYABUSA, Science, 312, 1341-1344

13. Yano, H., et al., 2006, Touch-down sites of the Hayabusa spacecraft at the Muses Sea area on asteroid 25143 Itokawa, Science, 312, 1350-1353 